\title{
A Debugging Scheme for Declarative Equation Based Modeling Languages
}

\author{
Peter Bunus and Peter Fritzson \\ Department of Computer and Information Science, Linköping University \\ SE 581-83, Linköping, Sweden \\ \{petbu,petfr\}@ida.liu.se
}

\begin{abstract}
This paper concerns the static analysis for debugging purposes of programs written in declarative equation based modeling languages. We first give an introduction to declarative equation based languages and the consequences equation based programming has for debugging. At the same time, we examine the particular debugging problems posed by Modelica, a declarative equation based modeling language. A brief overview of the Modelica language is also given. We also present our view of the issues and solutions based on a proposed framework for debugging declarative equation based languages. Program analysis solutions for program understanding and for static debugging of declarative equation based languages, based on bipartite graph decomposition, are presented in the paper. We also present an efficient way to annotate the underlying equations in order to help the implemented debugger to eliminate the heuristics involved in choosing the right error fixing solution. This also provides means to report the location of an error caught by the static analyzer or by the numeric solver, consistent with the user's perception of the source code and simulation model.
\end{abstract}

Keywords: Declarative equation based language, modeling languages, bipartite graphs, graph decomposition techniques, static analysis, debugging, Modelica

\section{Introduction}

Simulation models are increasingly being used in problem solving and in decision making since engineers need to analyze increasingly complex and heterogeneous physical systems. In order to support mathematical modeling and simulation, a number of object-oriented and/or declarative acausal modeling languages have emerged. The advantage of such a modeling language is that the user can concentrate on the logic of the problem rather than on a detailed algorithmic implementation of the simulation model.

Equation based declarative programming presents new challenges in the design of programming environments. In order for declarative equation based modeling languages to achieve widespread acceptance, associated programming environments and development tools must become more accessible to the user. 
A significant part of the simulation design effort is spent on detecting deviations from the specifications and subsequently localizing the sources of such errors. Employment of debugging environments that control the correctness of the developed source code has been an important factor in reducing the time and cost of software development in classical programming languages. Currently, few or no tools are available to assist developers debugging declarative equation based modeling languages. Since these languages usually are based on objectorientation and acausal physical modeling, traditional approaches to debugging are inadequate and inappropriate to solve the error location problem. To begin to address this need, we propose a methodology for implementing an efficient debugging framework for high level declarative equation based languages, by adapting graph decomposition techniques for reasoning about the underlying systems of equations. Detecting anomalies in the source code without actually solving the underlying system of equations provides a significant advantage: the modeling error can be corrected before embarking on a computationally expensive numerical solution process provided by a numerical solver. The errors detected by the numerical solvers are usually reported in a way which is not consistent with the user's perception of the declarative source code.

This paper is organized as follows: Section 2 provides a very brief description of Modelica, a declarative equation based modeling language. Section 3 give some explanations why is hard to debug declarative equation based languages and in Section 4 related work is briefly surveyed. In Section 5 a simple simulation model together with the underlying declarative specification is presented. Then we present several graph decomposition techniques and our algorithmic debugging approach based on those techniques. Section 7 provides some details about the structures used to annotate the underlying equations of the simulation model, in order to help the debugger to eliminate the heuristics when multiple choices are available to fix an error. In Section 8 explanations about debugging of an over-constrained system are given. Implementation details of the debugger are given in Section 9. Finally, Section 10 concludes and summarizes the work.

\section{Modelica, a Declarative Modeling Language}

Before describing the difficulties of debugging a declarative equation based language, we will acquaint the reader with Modelica, a declarative equation based modeling language. This part of the paper will briefly describe the Modelica language by presenting some language features which are necessary to understand the ideas presented in the paper. Modelica is a new language for hierarchical object-oriented physical modeling which is developed through an international effort $[10,7]$. The language unifies and generalizes previous object-oriented modeling languages. Modelica is intended to become a de facto standard. The language has been designed to allow tools to generate efficient simulation code automatically with the main objective to facilitate exchange of models, model libraries and simulation specifications. It allows defining simulation models in a declarative manner, modularly and hierarchically and combining various formalisms 
expressible in the more general Modelica formalism. The multidomain capability of Modelica gives the user the possibility to combine electrical, mechanical, hydraulic, thermodynamic, etc., model components within the same application model. Compared to other modeling languages available today, Modelica offers four important advantages from the simulation practitioners point of view:

- Acausal modeling based on ordinary differential equations (ODE) and differential algebraic equations (DAE). There is also ongoing research to include partial differential equations (PDE) in the language syntax and semantics [20].

- Multi-domain modeling capability, which gives the user the possibility to combine electrical, mechanical, thermodynamic, hydraulic etc., model components within the same application model.

- A general type system that unifies object-orientation, multiple inheritance, and generics templates within a single class construct. This facilitates reuse of components and evolution of models.

- A strong software component model, with constructs for creating and connecting components. Thus the language is ideally suited as an architectural description language for complex physical systems, and to some extent for software systems.

The reader of the paper is referred to [17,18] and [22] for a complete description of the language and its functionality from the perspective of the motivations and design goals of the researchers who developed it. Those interested in shorter overviews of the language may wish to consult [10] or [7].

\subsection{Modelica Acausal Modeling}

At the lowest level of the language equations are used to describe the relations between the quantities of a model. As it was mentioned before, one of the distinctive features of Modelica is the acausal programming model. The equations should be stated in a neutral form without consideration of order of elements evaluation in the model. Modelica computation semantics does not depend on the order in which equations are written. However, this property complicates the debugging process. The acausality makes Modelica library classes more reusable than traditional classes containing assignment statements where the input-output causality is fixed, since Modelica classes adapt to the data flow context in which they are used. The data flow context is defined by telling which variables are needed as outputs and which are external inputs to the simulated system. From the simulation practice point of view this generalization enables both simpler models and more efficient simulation. The declarative form allows a one-to-one correspondence between physical components and their software representation.

\subsection{Modelica Classes}

Modelica programs are built from classes, like in other object-oriented languages. The main difference compared with traditional object-oriented languages is that 


\subsection{Modelica Connections and Connectors}

Equations in Modelica can also be specified by using the connect statement. The statement connect (v1, v2) expresses coupling between variables. These variables are called connectors and belong to the connected objects. Connections specify interaction between components. A connector should contain all quantities needed to describe the interaction. This gives a flexible way of specifying topology of physical systems described in an object-oriented way using Modelica.

For example, Pin is a connector class that can be used to specify the external interfaces for electrical components that have pins. Each Pin is characterized by two variables: voltage $\mathrm{v}$ and current $\mathrm{i}$. A connector class is defined as follows:

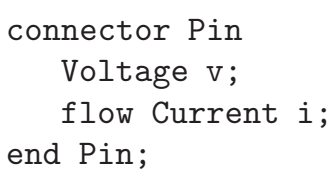

The flow prefix is required for variables which belong to instances of connector classes and specify flow quantities, e.g. current flow, fluid flow, force, etc. Such quantities obey Kirchhoff's current law of summing all flows into a specific point to zero. Connection statements are used to connect instances of connection classes. A connection statement connect(Pin1,Pin2), with Pin1 and Pin2 of connector class Pin, connects the two pins so that they form one node. This implies two equations, namely:

Pin1.v $=$ Pin2.v; Pin1.i + Pin2.i $=0$

\section{Arising Difficulties when Debugging Declarative Equation Based Languages}

The application of algorithmic debugging techniques [21] and generalized algorithmic debugging techniques [11] to the evaluation of structural procedural languages is an approach which has found increased applicability over the past years. However, traditional approaches to debugging are inadequate and inappropriate to solve the error location problem in declarative equation based languages. The fundamental problem is that conventional debuggers and debugging techniques are based on observation of execution events as they occur.

Even nontraditional declarative debugging techniques such as the abovementioned algorithmic debugging method, are inadequate for equation-based languages. In order to see this, consider invoking an algorithmic program debugger [22] on a functional/logic program after noticing an external symptom of a bug. The debugger executes the program and builds a trace execution tree at the function level while saving some useful trace information such as function names and input/output parameter values. In other words, the algorithmic debugging method is dependent on the use of functions and function calls in the language. In the case of declarative equation based languages, there is no 
clear execution tree and the inputs and outputs are not clearly stated inside the model. In conclusion we can take a look on why errors are hard to find in a declarative equation based language:

- There is a cause-effect gap between the time or space when an error occurs and the time or space when the error becomes apparent to the programmer.

- The acausality of the language eliminates the use of program traces as a debugging guide.

- The transformation process from the declarative form to the procedural form looses or obscures a lot of model structure information, which might be useful for debugging purposes.

- Even in a highly structured system, which extensively uses hierarchical and modular simulation models, surprising events may occur because the human mind is not able to fully comprehend the many conditions that can arise mainly because of the interactions of the components.

- Debugging is in some sense harder to perform because much run-time debugging must be replaced by compile-time static checking.

- The static analysis is mostly global and it is necessary to consider the whole program.

\section{Related Work}

Our debugging approach follows the same philosophy as does the reduction of constraint systems used for geometric modeling in [2]. In [2] the resulting algebraic equations from a geometric modeling by constraints problem are decomposed in well constrained, over and under constrained subsystems for debugging purposes. The well constrained systems are further decomposed into irreductible subsystems for speeding up the resolution in case of reductible systems.

In [4] attention is paid to the well-constrained part of the equations by proposing new algorithms for solving the structurally well-constrained problems by combining the use of numerical solvers with intelligent backtracking techniques. The backtracking of the ordered blocks is performed when a block has no solution. This approach mostly deals with so called numerical problems, which of course are due to erroneous modeling and wrong declarative specification of the problem, and requires the use of numerical solvers. In [4] an algorithm for underconstrained problems is presented which deals with the problem of selecting the input parameters that leads to a good decomposition.

Our work and the above-mentioned related work share the common goal of providing users with an effective way of debugging constraint related problems. The above-presented related work, is extended by our approach by incorporating the ordinary and differential algebraic equations into the static analysis by manipulating the system of equations to achieve an acceptable index [19] and linking the graph decomposition techniques and algorithms to the original source code of the declarative equational based language. To our knowledge, no existing simulation system which employ a declarative equation based modeling language performs an efficient mapping between information obtained from graph decomposition techniques and the original program source code. 


\section{Simulation Model Example}

Obviously, each simulation problem is associated with a corresponding mathematical model. In dynamic continuous simulation the mathematical model is usually represented by a mixed set of algebraic equations and ordinary differential equations. For some complicated simulation problem the model can be represented by a mixed set of ordinary differential equations (ODEs), differential algebraic equations (DAEs) and partial differential equations (PDEs). Simulation models can become quite large and very complex in their structure sometimes involving several thousand equations.

The system of equations describing the overall model is obtained by merging the equations of all simple models and all binding equations generated by the connect statements. In Fig.1 the Modelica source code of a simple simulation model consisting of a resistor connected in parallel to sinusoidal voltage is given. The intermediate form is also given for explanatory purposes. The Circuit model is represented as an aggregation of the Resistor, Source and Ground submodels connected together by means of physical ports.

\section{Graph Based Representation of the Underlying Model}

Many practical problems form a model of interaction between two different types of objects and can be phrased in terms of problems on bipartite graphs. The expressiveness of the bipartite graphs in concrete practical applications has been demonstrated many times in the literature [5,3]. We will show that the bipartite graph representations are general enough to efficiently accommodate several numeric analysis methods in order to reason about the solvability and unsolvability of the flattened system of equations and implicitly about the simulation model behavior. Another advantage of using the bipartite graphs is that it offers an efficient abstraction necessary for program transformation visualization when the equation based declarative specifications are translated to procedural form.

The bipartite graph representation and the associated decomposition techniques are widely used internally by compilers when generating the procedural form from the declarative equation based description of the simulation model $[8,16]$ but none of the existing simulation systems use them for debugging purposes or expose them visually for program understanding purposes.

In the remaining of this paragraph it is our intention to give the basic definitions and some of the notation which we shall use throughout the rest of this paper.

Definition 1: A bipartite graph is an ordered triple $G=\left(V_{1}, V_{2}, E\right)$ such that $V_{1}$ and $V_{2}$ are sets, $V_{1} \cap V_{2}=\emptyset$ and $E \subseteq\left\{\{x, y\} ; x \in V_{1}, y \in V_{2}\right\}$. The vertices of $G$ are elements of $V_{1} \cap V_{2}$. The edges of $G$ are elements of $E$.

Definition 2: Let $G$ be a graph with vertex set $V(G)=\left\{v_{1}, v_{2}, \ldots, v_{p}\right\}$ and edge set $E(G)=\left\{e_{1}, e_{2}, \ldots, e_{q}\right\}$. The incidence matrix of $G$ is the $p \times q$ matrix $M(G)=\left|m_{i j}\right|$, where $m_{i j}$ is 1 if the edge $e_{i j}$ is incident with vertex $v_{i j}$ and 0 otherwise. 


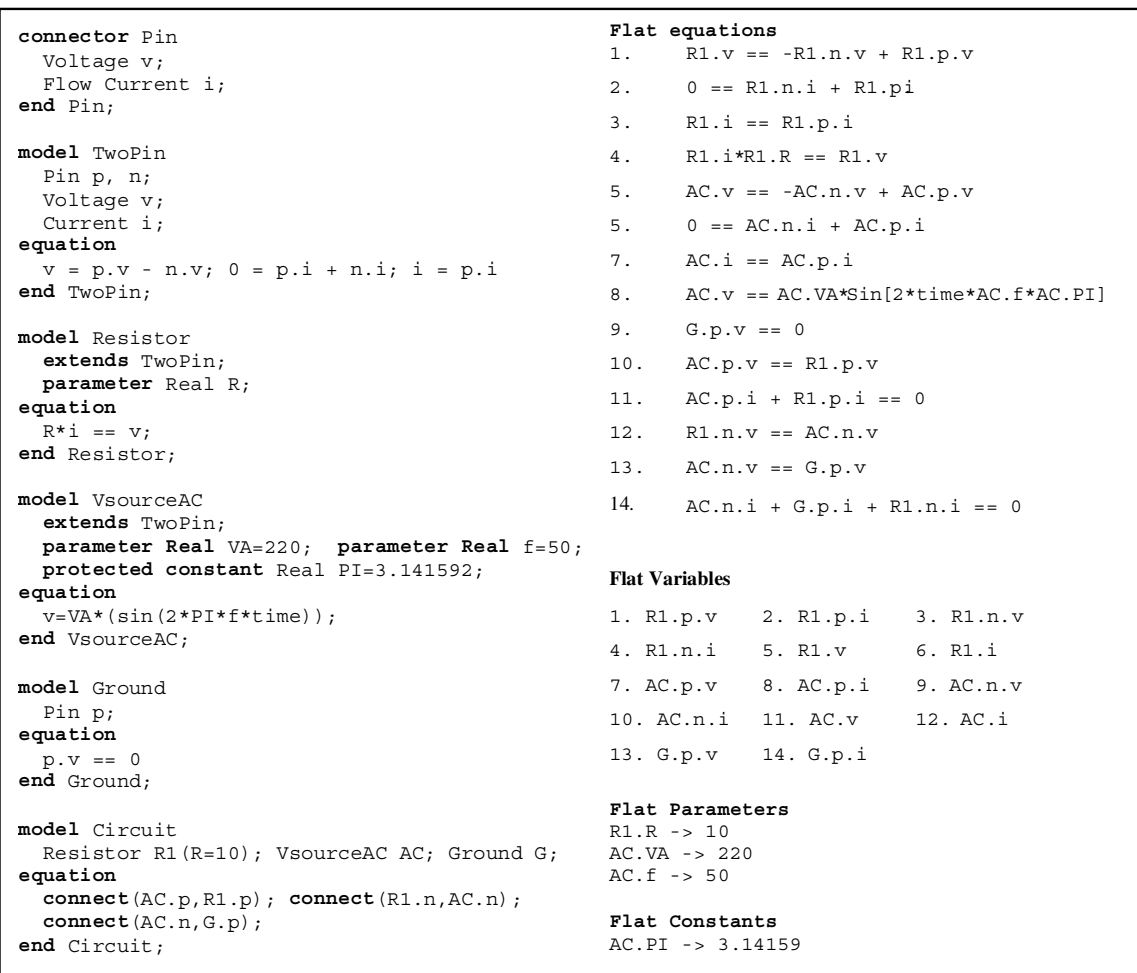

Fig. 1. Modelica source code of a simple simulation model and the corresponding flattened systems of equation, variables, parameters and constants

We consider the bipartite graph associated to a given system of equations resulting from the flattening operation of the declarative specification. Let be $V_{1}$ the set of equations and $V_{2}$ the set of variables representing unknowns. An edge between $e q \in V_{1}$ and var $\in V_{2}$ means that the variable var appears in the corresponding equation eq. Based on this rule the associated bipartite graph of the flattened system of equation from Fig. 1 is presented in Fig.2.

\subsection{Bipartite Matching Algorithms}

The following definitions are given:

Definition 3: A matching is a set of edges from graph $G$ where no two edges have a common end vertex.

Definition 4: A maximum matching is the matching with the largest possible number of edges.

Definition 5: A matching $M$ of a graph $G$ is maximal if it is not properly contained in any other matching. 


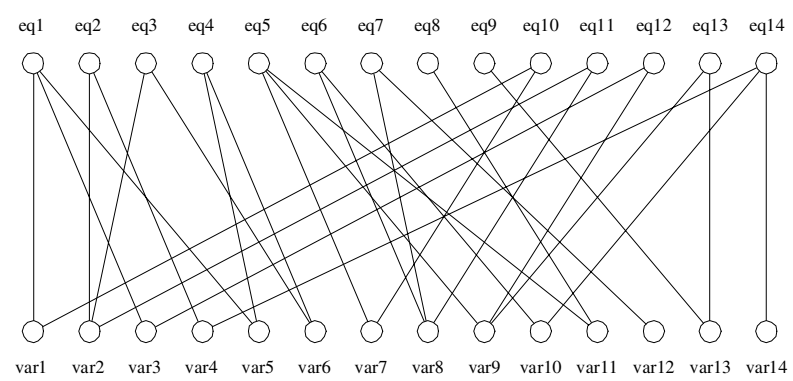

Fig. 2. The associated bipartite graph of the simple circuit model from Fig.1

Definition 6: A vertex $v$ is saturated or covered by a matching $M$ if some edge of $M$ is incident with $v$. An unsaturated vertex is called a free vertex.

Definition 7: A perfect matching $P$ is a matching in a graph $G$ that covers all its vertices.

In Fig. 3 all the possible perfect matchings of a simple bipartite graph are presented. It should be noted that a maximum matching and the perfect matching of a given bipartite graph is not unique.
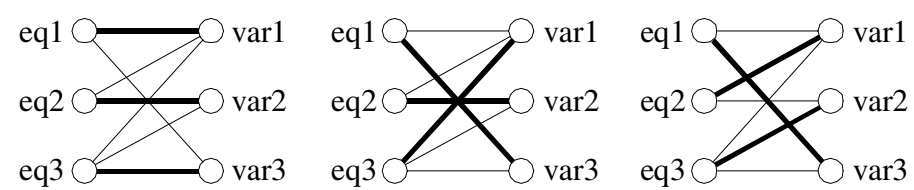

Fig. 3. An example bipartite graph with all the possible perfect matchings marked by thick lines

The equation system associated with a perfect matching is structurally wellconstrained and therefore can be further decomposed into smaller blocks and sent to a numerical solver. Fig.4 illustrates the maximal matching of the associated bipartite graph to the simulation model presented in Fig.1. It is worth noting that, in this case, the maximal matching is also a perfect matching of the associated bipartite graph.

From the computational complexity point of view, the best sequential algorithm for finding a maximum matching in bipartite graphs is due to Hopcroft and Karp [13]. The algorithm solves the maximum cardinality matching problem in $O\left(n^{\frac{5}{2}}\right)$ time and $O(n m)$ memory storage where $n$ is the number of vertices and $m$ is the number of edges. 


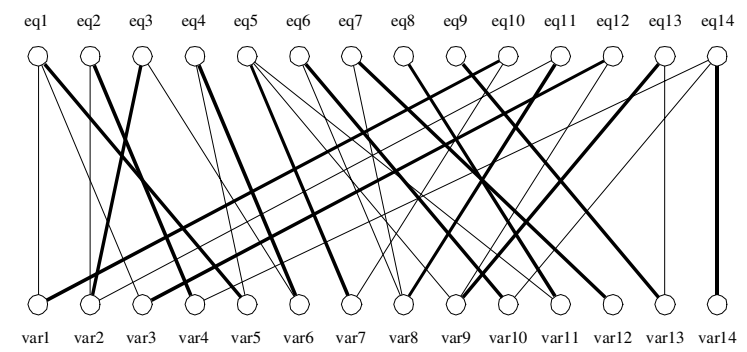

Fig. 4. One possible perfect matching of the simulation model associated bipartite graph

\subsection{Dulmage - Mendelsohn's Canonical Decomposition}

In this section we shall present a structural decomposition of a bipartite graph associated with a simulation model which relies on the above presented vertex coverings. The algorithm is due to Dulmage and Mendelsohn [6] and canonically decompose any maximum matching of a bipartite graph in three distinct parts: over-constrained, under-constrained, and well-constrained part.

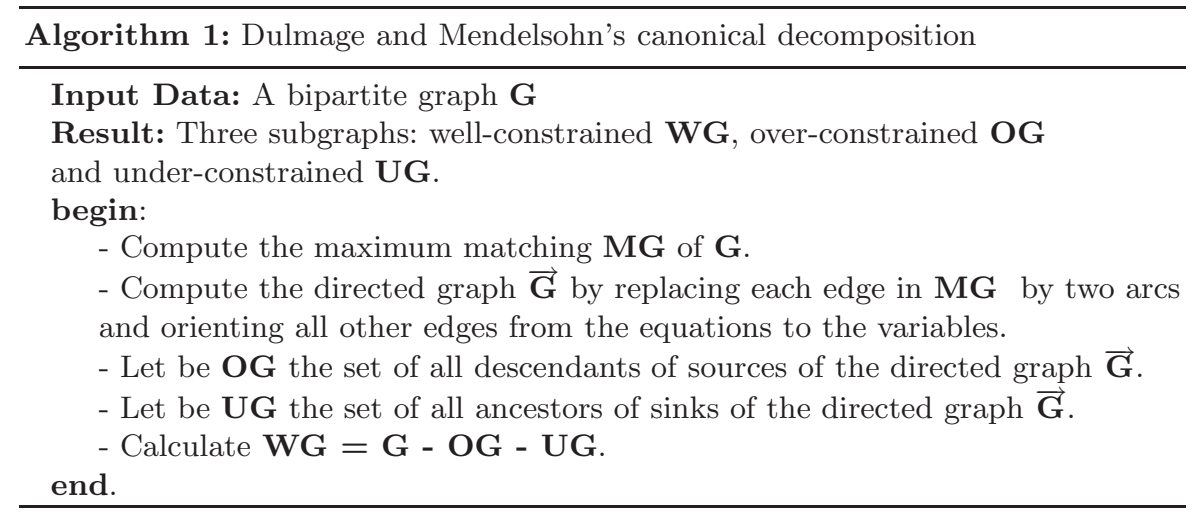

The over-constrained part: the number of equations in the system is greater than the number of variables. The additional equations are either redundant or contradictory and thus yield no solution. A possible error fixing strategy is to remove the additional over-constraining equations from the system in order to make the system well-constrained. Even if the additional equations are soft constraints which means that they verify the solution of the equation system and are just redundant equations, they are reported as errors by the debugger because there is no way to verify the equation solution during static analysis without explicitly solving them. 
The under-constrained part: the number of variables in the system is greater than the number of equations. A possible error fixing strategy would be to initialize some of the variables in order to obtain a well-constrained part or add additional equations to the system.

Over and under-constrained situations can coexist in the same model. In the case of over-constrained model, the user would like to remove the overconstraining equations in a manner which is consistent to the original source code specifications, in order to alleviate the model definition.

The well-constrained part: the number of equations in the system is equal to the number of variables and therefore the mathematical system of equations is structurally sound having a finite number of solutions. This part can be further decomposed into smaller solution subsets. A failure in decomposing the wellconstrained part into smaller subsets means that this part cannot be decomposed and has to be solved as it is. A failure in numerically solving the well-constrained part means that no valid solution exists and there is somewhere a numerical redundancy in the system.

The decomposition captures one of the many possible solutions in which the model can be made consistent. The direct solution proposed by the decomposition sometimes cannot be acceptable from the restriction imposed by the modeling language or by the modeling methodology by itself. Therefore a search through the equivalent solution space needs to be done and, check whether the equivalent solutions are acceptable.

\section{Equation Annotations}

For annotating the equations we use a structure which resembles the one developed in [9]. We define an annotated equation as a record with the following structure: 〈Equations, Name, Description, No. of associated eqs., Class name, Flexibility level, Connector generated $\rangle$. The values defined by annotations are later incorporated in the error repair strategies, when heuristics involved in choosing the right option from a series of repair strategies needs to be eliminated.

Table 1. An example of an annotated equation

\begin{tabular}{ll}
\hline Attribute & Value \\
\hline Equation & $\mathrm{R} 1 . \mathrm{i} * \mathrm{R} 1 . \mathrm{R}=\mathrm{R} 1 . \mathrm{v}$ \\
Name & "eq4" \\
Description & "Ohm's Law for the resistor component" \\
No. of associated eqs & 1 \\
Class Name & "Resistor" \\
Flexibility Level & 3 \\
Connector generated & no \\
\hline
\end{tabular}


The Class Name tells from which class the equation is coming. This annotation is extremely useful in exactly locating the associated class of the equation and therefore providing concise error messages to the user.

The No. of associated eqs. parameter specify the number of equations which are specified together with the annotated equation. In the above example the No. of associated eqs. is equal to one since there are no additional equations specified in the Resistor component. In the case of the TwoPin component the number of associated equations is equal to 3 . If one associated equation of the component need to be eliminated the value is decremented by 1 . If, during debugging, the equation $\mathrm{R} 1 . \mathrm{i} * \mathrm{R} 1 . \mathrm{R}=\mathrm{R} 1 . \mathrm{v}$ is diagnosed to be an over-constraining equation and therefore need to be eliminated, the elimination is not possible because the model will be invalidated in that way (the No. of associated eqs. cannot be equal to 0 ) and therefore other solutions need to be taken into account.

The flexibility level, in a similar way as it is defined in [10], allows the ranking of the relative importance of the constraint in the overall flattened system of equations. The value can be in the range of 1 to 3 , with 1 representing the most rigid equation and 3 being the most flexible equation. Equations, which are coming from a partial model and therefore are inherited by the final model, have a greater rigidity compared to the equations defined in the final model. For example, in practice, it turns out that the equations generated by connections are more rigid from the constraint relaxation point of view than the equations specified inside the model. Taking into account these formal rules, a maximal flexibility level will be assigned for an equation defined inside a final Modelica class. In conclusion a maximum flexibility level will be defined for the equations in the final model, followed by equations defined in partial classes and equations generated by the connect statements.

The Connector generated is a Boolean attribute which tells whether the equation is generated or not by a connect statement. Usually these equations have a very low flexibility level.

It is worth nothing that the annotation attributes are automatically initialized by the static analyzer, incorporated in the front end of the compiler, by using several graph representations [12] of the declarative program code.

\section{Debugging of an Over-Constrained System}

Let us again examine the simple simulation example presented in Fig.1 where an additional equation ( $i=23$ ) was intentionally introduced inside the Resistor component in order to obtain a generally over-constrained system. The D\&M canonical decomposition will lead to two parts: a well-constrained part and an over-constrained part (see Fig. 5.). Equation "eq11" is a non-saturated vertex of the equation set so it is a source for the over-constrained part. Starting from "eq11" the directed graph can be redrawn as is illustrated in Fig.6. An immediate solution of fixing the over-constrained part is to eliminate "eq11" which will lead to a well-constrained part and therefore the equation system becomes structurally sound. However, examining the associated annotations to the "eq11": 
$\langle A C \cdot p . v==$ R1.pv, "eq11", " ", 2, "Circuit", 1, yes $\rangle$, one can note that the equation is generated by a connect statement from the Circuit model and the only way to remove the equation is to remove the connect (AC.p, R1.p) statement. But removing the above-mentioned statement will remove two equations from the flattened model, which is indicated by the No. of associated eqs. = 2 parameter. One should also note the flexibility level of the equation is equal to 1 , which is extremely low, indicating that the equation is extremely rigid. Therefore an another solution need to be found, namely another equation need to be eliminated from the equation system instead of removing the equation $\mathrm{AC} \cdot \mathrm{p} \cdot \mathrm{v}==\mathrm{R} 1 \cdot \mathrm{pv}$.

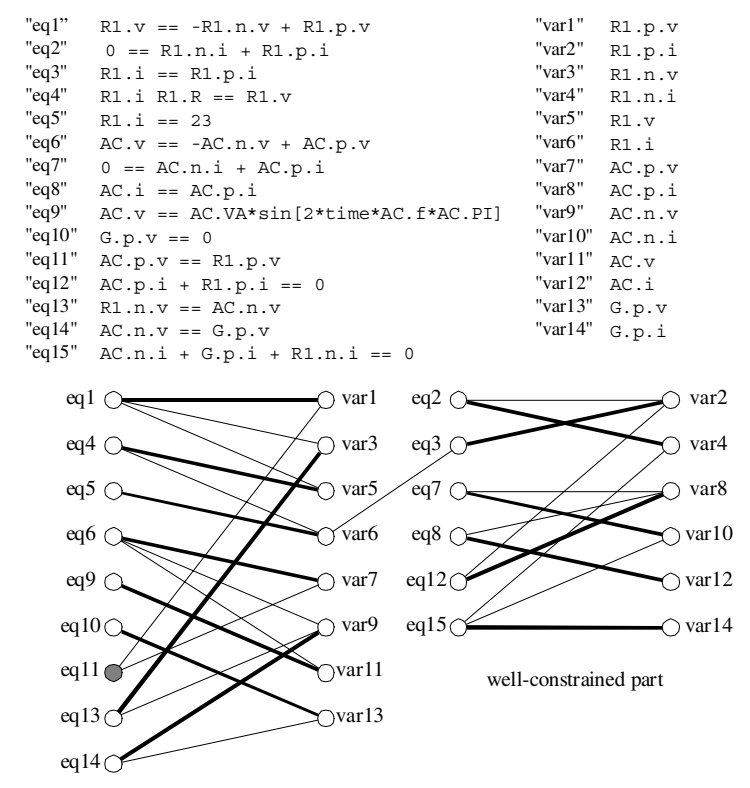

over-constrained part

Fig. 5. The decomposition of an over-constrained system

In the next step of the debugging procedure for the over-constrained system of equations we need to introduce several definitions regarding some particular equation subsets which have special properties from the structural analysis point of view.

Definition 8: We call the equivalent over-constraining equation list associated to a system of equations the list of equations $\left\{e q_{1}, e q_{2}, \ldots, e q_{n}\right\}$ from where eliminating any of the component equations will lead to a well constrained system of equations. 
Definition 9: We call the reduced equivalent over-constraining equation list the subset of equations obtained from the equivalent over-constraining equations after the language constraints have been applied.

When the size of the reduced equivalent over-constraining equation list exceeds 1 , the automatic debugging is no longer available, and then the list should be output to the user by the debugger in order to solve the conflicting situation.

From the over-constrained part resulting from the D\&M decomposition we can construct an algorithm to find the equivalent over-constraining list based on the associated directed graph of the over-constrained part. We describe the algorithm as follows:

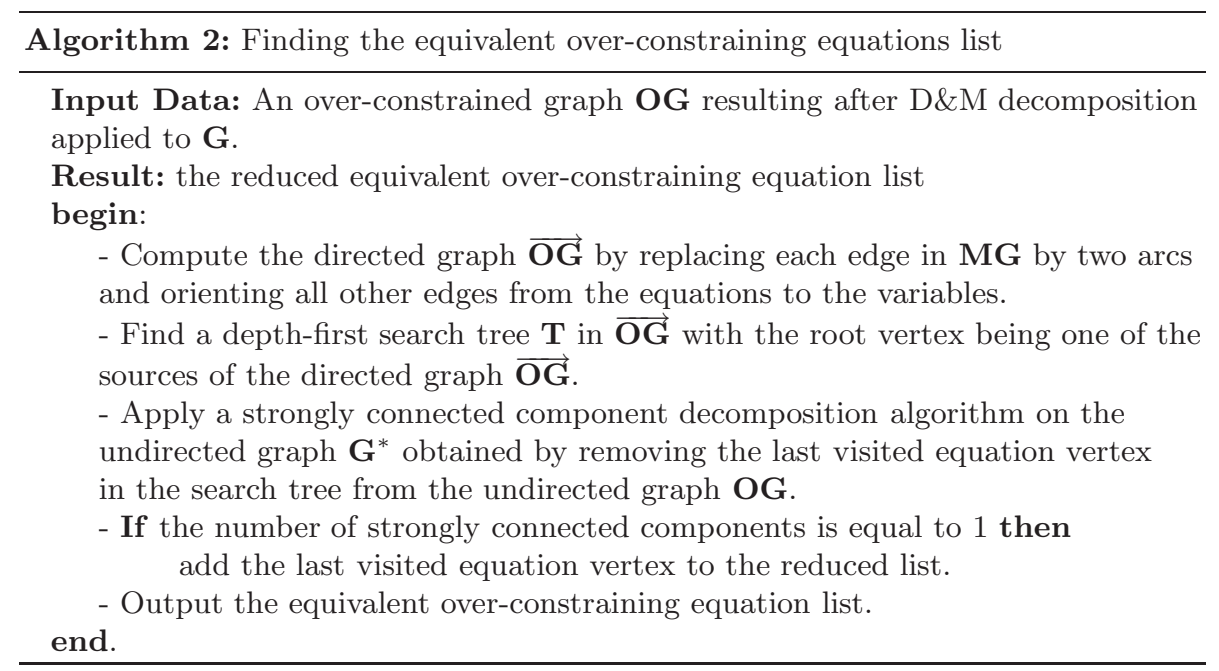

An algorithm for computing the reduced equivalent over-constraining equation list is given below:

If the length of the reduced equivalent over-constraining list is equal to 1 automatic debugging of the model is possible by eliminating the equation from the simulation model without any supplementary user-intervention. Of course the history list together with the elimination is output to the user. If the length of the list is greater than 1, this means that several error fixing strategies are possible and therefore user intervention is required. The reduced list is output to the user starting with the equation which has the higher flexibility level.

In our case the set of equivalent over-constraining equations is "eq11", "eq13", "eq10", "eq5", "eq9"\}. "Eq11" was already analyzed and therefore can be eliminated from the set. "Eq13" is eliminated too for the same reasons as equation "eq11". Analyzing the remaining equations $\{" e q 10 ", " e q 5 ", " e q 9 "\}$ one should note that they have the same flexibility level and therefore they are candidates for elimination with an equal chance. But analyzing the value of the No. of associated eqs. parameter, equation "eq10" and "eq9" have that at- 


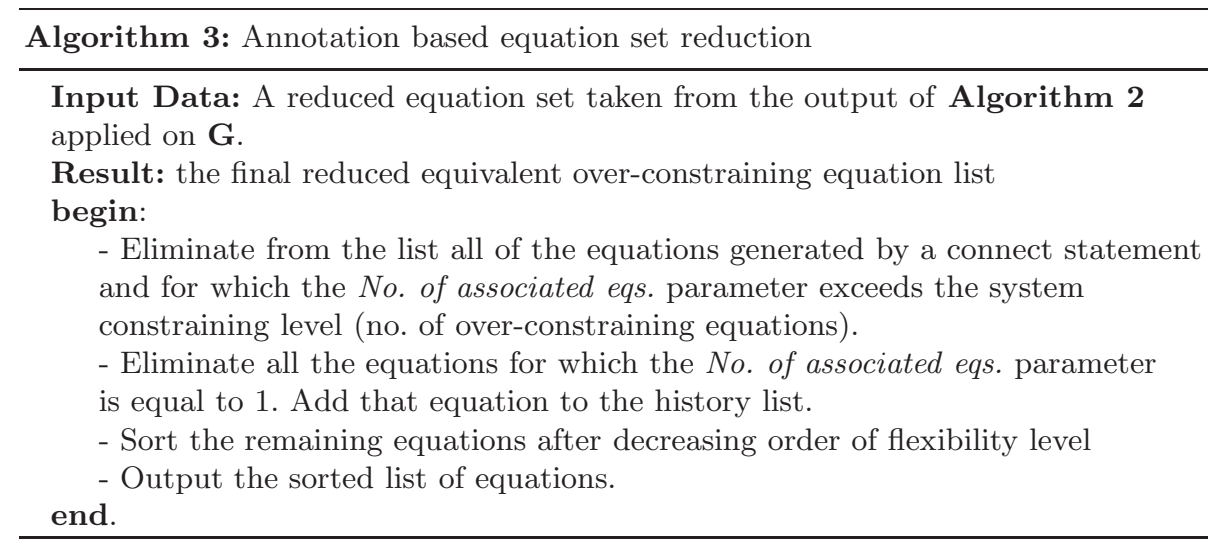

tribute equal to one, which means that they are singular equations defined inside the model. Eliminating one of these equations will invalidate the corresponding model, which is probably not the intention of the modeler. Examining the annotations corresponding to equation "eq5" one can see that it can be safely eliminated because the flexibility level is high and eliminating the equation will not invalidate the model since there is another equation defined inside the model. After choosing the right equation for elimination the debugger tries to identify the associated class of that equation based on the Class name parameter defined in the annotation structure. Having the class name and the intermediate equation form (R1.i=23) the original equation can be reconstructed ( $i=23)$ indicating exactly to the user which equation need to be removed in order to make the simulation model mathematically sound. In that case the debugger has correctly located the faulty equation in the simulation system which was previously introduced by us.

In conclusion, by examining the annotations corresponding to the set of equations which need to be eliminated, the implemented debugger can automatically determine the possible error fixing solutions and of course prioritize them. For example, by examining the flexibility level of the associated equation compared to the flexibility level of another equation the debugger can prioritize the proposed error fixing schemes. When multiple valid error fixing solutions are possible and the debugger cannot decide which one to chose, a prioritized list of possible error fixes is presented to the user for further analysis and decision. In those cases, the final decision must be taken by the user, as the debugger cannot know or doesn't have sufficient information to decide which equation is over-constraining. The advantage of this approach is that the debugger automatically identifies and solves several anomalies in the declarative simulation model specification without having to execute the system. 


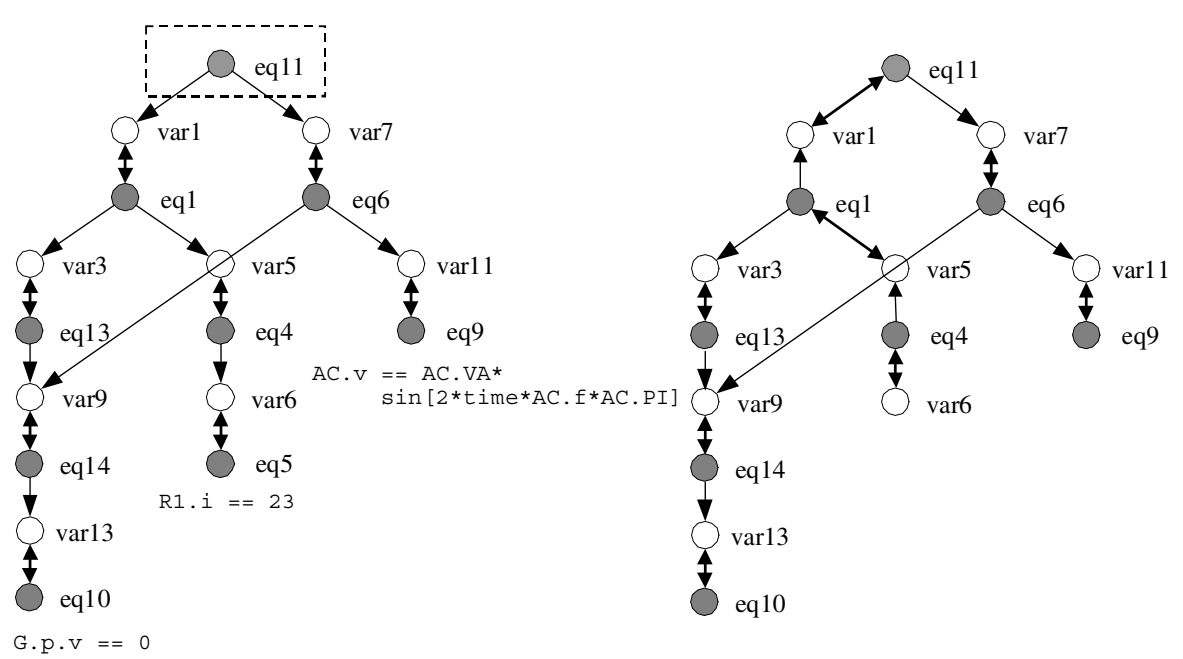

Fig. 6. An associated directed graph to the over-constrained part starting from "eq11" (left) and the fixed well-constrained directed graph by eliminating equation "eq5" (right)

\section{Prototype Debugger Implementation Details}

For the above presented graph decomposition techniques to be useful in practice, we must be able to construct and manage the graph representation of the declarative specification efficiently. Another important factor which must to be taken into account is the incrementality of the approach in order to accommodate incremental static analyzers to be added to the existing simulation environment of the declarative equation based language. In this section, we outline the architecture and organization of the implemented debugger attached to the simulation environment.

A prototype debugger was built and attached to the MathModelica simulation environment as a testbed for evaluating the usability of the above presented graph decomposition techniques for debugging declarative equation based languages. MathModelica is an integrated problem-solving environment (PSE) for full system modeling and simulation $[14,15]$. The environment integrates Modelica-based modeling and simulation with graphic design, advanced scripting facilities, integration of code and documentation, and symbolic formula manipulation provided via Mathematica. Import and export of Modelica code between internal structured and external textual representation is supported by MathModelica. The environment extensively supports the principles of literate programming and integrates most activities needed in simulation design: modeling, documentation, symbolic processing, transformation and formula manipulation, input and output data visualization. 
As indicated previously, it is necessary for the compiler to annotate the underlying equations to help identify the equations and to help eliminating the heuristic involved in choosing the right solution. Accordingly, we modified the front end of the compiler to annotate the intermediate representation of the source code where equations are involved. The annotations are propagated appropriately through the various phases of the compiler, and, when an error is detected, the debugger uses them to eliminate some of the heuristics involved in the error solving process and, of course, to exactly identify the problematic equations and to generate error messages consistent with the user's perception of the source code corresponding to the simulation model. The debugger focuses on those errors whose identification would not require the solution of the underlying system of equations.

\section{Summary and Conclusion}

The acausality of declarative equation based languages makes many program errors hard to find. Often the error messages do not refer back to the component of the model which is the cause of the problem. The situation if further complicated by program optimizations on the source code, which eliminates or obscures a lot of the model structure information, which are useful for debugging purposes.

In this paper we have presented a general framework for debugging declarative equation based languages. The contributions of this paper are twofold: the proposal of integrating graph decomposition techniques for debugging declarative equation based languages and an efficient equation annotation structure which helps the debugger to eliminate some of the heuristics involved in the error solving process. The annotations also provides an efficient way of identifying the equations and therefore helps the debugger in providing error messages consistent with the user's perception of the original source code and simulation model. The implemented debugger helps to statically detect a broad range of errors without having to execute the simulation model. Since the simulation system execution is expensive the implemented debugger helps to greatly reduce the number of test cases used to validate the simulation model.

One extended case study, along with a number of small examples scattered throughout this paper, illustrates the main points and potential applications of the graph theory related to the proposed method for debugging of declarative equation based languages.

\section{Acknowledgements}

We thank Peter Aronsson for his contributions to the implementation of the MathModelica interpreter and the entire MathModelica team, without which this work would not have been possible. The work has been supported by MathCore $A B$ and by the ECSEL Graduate School supported by the Swedish Strategic Research Foundation. 


\section{References}

1. Abadi M., and Cardelli: A Theory of Objects. Springer Verlag, ISBN 0-387-94775-2, 1996. 283

2. Ait-Aoudia, S.; Jegou, R. and Michelucci, D. "Reduction of Constraint Systems." In Compugraphic, pages 83-92, Alvor, Portugal, 1993. 285

3. Asratian A. S.; Denley T. and Häggkvist R. Bipartite Graphs and their Applications. Cambridge University Press 1998. 286

4. Bliek, C.; Neveu, B. and Trombettoni G. "Using Graph Decomposition for Solving Continuous CSPs", Priciples and Practice of Constraint Programming, CP'98, Springer LNCS 1520, pages 102-116, Pisa, Italy, November 1998. 285

5. Dolan A. and Aldous J. Networks and algorithms - An introductory approach. John Wiley and Sons 1993 England. 286

6. Dulmage, A. L., Mendelsohn, N. S. Coverings of bipartite graphs, Canadian J. Math., 10, 517-534. 289

7. Elmqvist, H.; Mattsson S.E and Otter, M. "Modelica - A Language for Physical System Modeling, Visualization and Interaction." In Proceedings of the 1999 IEEE Symposium on Computer-Aided Control System Design (Hawaii, Aug. 22-27) 1999. 281, 282

8. Elmqvist, H. A Structured Model Language for Large Continuous Systems. PhD thesis TFRT-1015, Department of Automatic Control, Lund Institute of Technology, Lund, Sweden. 1978. 286

9. Flannery, L. M. and Gonzalez, A. J. Detecting Anomalies in Constraint-based Systems, Engineering Applications of Artificial Intelligence, Vol. 10, No. 3, June 1997, pages. 257-268. 290

10. Fritzson, P. and Engelson, V. "Modelica - A Unified Object-Oriented Language for System Modeling and Simulation." In Proceedings of the 12th European Conference on Object-Oriented Programming (ECOOP'98 , Brussels, Belgium, Jul. 20-24), 1998. 281, 282

11. Fritzson, P.; Shahmehri, N.; Kamkar, M. and Gyimothy, T. Generalized algorithmic debugging and testing. ACM Letters on Programming Languages and Systems, 1(4):303-322, December 1992. 284

12. Harrold, M.J and Rothermel, G. "A Coherent Family of Analyzable Graphical Representations for Object-Oriented Software." Technical Report OSU-CISRC-11/96TR60, November, 1996, Department of Computer and Information Science Ohio State University 291

13. Hopcroft, J. E. and Karp, R. M. An $n^{\frac{5}{2}}$ algorithm for maximum matchings in bipartite graphs. SIAM Journal of Computing, 2(4):225-231, December 1973. 288

14. Jirstrand, M. "MathModelica - A Full System Simulation Tool". In Proceedings of Modelica Workshop 2000 (Lund, Sweden, Oct. 23-24), 2000. 295

15. Jirstrand, M.; Gunnarsson J. and Fritzson P. "MathModelica - a new modeling and simulation environment for Modelica. "In Proceedings of the Third International Mathematica Symposium (IMS'99, Linz, Austria, Aug), 1999. 295

16. Maffezzoni C.; Girelli R. and Lluka P. Generating efficient computational procedures from declarative models. Simulation Practice and Theory 4 (1996) pages 303-317. 286

17. Modelica Association. Modelica - A Unified Object-Oriented Language for Physical Systems Modeling - Tutorial and Design Rationale Version 1.4 (December 15, 2000). 282, 283 
18. Modelica Association. Modelica - A Unified Object-Oriented Language for Physical Systems Modeling - Language Specification Version 1.4. (December 15, 2000). 282

19. Pantelides, C. The consistent initialization of differentialalgebraic systems. SIAM Journal on Scientific and Statistical Computing, 9(2):213-231, March 1988. 285

20. Saldamli, L.; Fritzson, P. "Object-oriented Modeling With Partial Differential Equations". In Proceedings of Modelica Workshop 2000 (Lund, Sweden, Oct. 2324), 2000. 282

21. Shapiro Ehud Y. Algorithmic Program Debugging. MIT Press (May). 1982. 284

22. Tiller M. Michael. Introduction to Physical Modeling with Modelica. Kluwer Academic Publisher 2001. 282 